\title{
Telomere length differences between colorectal polyp subtypes: a colonoscopy- based case-control study
}

\author{
Sheetal Hardikar ${ }^{1,2^{*}}$ (D, Andrea N. Burnett-Hartmann ${ }^{2,3}$, Amanda I. Phipps ${ }^{2,4}$, Melissa P. Upton ${ }^{5}$, Lee-Ching Zhu ${ }^{6}$
} and Polly A. Newcomb $b^{2,4}$

\begin{abstract}
Background: Short telomeres have been associated with increased risk of many cancers, particularly cancers of the gastrointestinal tract including esophagus and stomach. However, the association between telomere length (TL) and colorectal cancer and its precursors, colorectal polyps, is not clear.

Methods: We investigated the relationship between $T L$ and risk of colorectal polyp subtypes in a colonoscopybased study in western Washington. Participants were 35-79 year-old enrollees at an integrated health care system, who underwent a colonoscopy between 1998 and 2007 ( $n=190)$, completed a self-administered questionnaire, provided blood samples, and were distinguished as having adenomas, serrated polyps, or as polyp-free controls through a standardized pathology review. Telomere length (T) relative to a single copy gene (S) was measured in circulating leukocytes from stored buffy coat samples using quantitative polymerase chain reaction. Multivariable polytomous logistic regression was used to compare case groups with polyp-free controls and other case groups; adjusted odds ratios (OR) and 95\% confidence intervals (CI) were estimated.

Results: TL in the shortest tertile (T/S ratio $<0.58)$ was associated with increased risk of adenomas and serrated polyps [OR $(95 \% \mathrm{Cl})$ were $1.77(0.81-3.88)$ and $2.98(1.15-7.77)$, respectively). When evaluated by lesion severity within each pathway, short TL was more strongly associated with advanced adenomas and sessile serrated polyps [OR $(95 \% \mathrm{Cl})=1.90(0.76-4.73)$ and 3.82(0.86-16.86), respectively], although the associations were not statistically significant.

Conclusions: Our results suggest that short TL may be associated with an increased risk of colorectal polyps in both the adenoma-carcinoma and serrated pathways. The risk was particularly notable for sessile serrated polyps, although the association was not statistically significant and sample size was limited.
\end{abstract}

Keywords: Adenomas, Serrated polyps, Sessile serrated polyps, Advanced adenomas, Telomere length

\section{Background}

Colorectal cancer (CRC) is a multi-pathway disease; unique CRC pathways are associated with distinct polyp precursor lesions. Advanced adenomas are known precursor lesions to CRC within the adenoma-carcinoma pathway, while sessile serrated adenomas/polyps (SSA/Ps), are precursors on the serrated pathway [1]. Currently, colorectal polyps are not diagnosed until colonoscopy, an

\footnotetext{
* Correspondence: sheetal.hardikar@hci.utah.edu

${ }^{1}$ Huntsman Cancer Institute, University of Utah, 2000 Circle of Hope Dr.

Room 4711, Salt Lake City, UT 84112, USA

${ }^{2}$ Public Health Sciences Division, Fred Hutchinson Cancer Research Center,

Seattle, WA, USA

Full list of author information is available at the end of the article
}

expensive and invasive procedure. Moreover, advanced adenomas are diagnosed in about $6 \%$ of those undergoing colonoscopy; rate of SSA/P diagnosis is lower [2]. If reliable biomarkers that enable risk stratification to identify those at highest risk for progression to CRC can be identified, patients could be triaged to tailored screening and surveillance regimes that coincide with their level of risk.

One such potential biomarker of interest is telomere length (TL). Telomeres, the DNA repeat sequences at ends of chromosomes, play an important role in maintaining genomic integrity [3]. Short TL has been evaluated as a biomarker for ageing, and age-related conditions, including

(c) The Author(s). 2018 Open Access This article is distributed under the terms of the Creative Commons Attribution 4.0 International License (http://creativecommons.org/licenses/by/4.0/), which permits unrestricted use, distribution, and reproduction in any medium, provided you give appropriate credit to the original author(s) and the source, provide a link to the Creative Commons license, and indicate if changes were made. The Creative Commons Public Domain Dedication waiver (http://creativecommons.org/publicdomain/zero/1.0/) applies to the data made available in this article, unless otherwise stated. 
cancers; particularly of the gastrointestinal tract [4]. The relationship between shortened telomeres and risk of sporadic colorectal cancers, however, is not yet clear. Here, we evaluate the association between TL and the risk for colorectal polyp subtypes.

\section{Methods}

\section{Study population}

The study population for the current project was derived from a parent study within Kaiser Permanente Washington, a large integrated healthcare provider in western Washington state. Details of the parent study have been previously published [5]. We selected 35-79 year old enrollees who had undergone an index colonoscopy for any indication from 1998 to 2003 (phase 1) and 20042007 (phase 2), and had available stored genomic DNA $(N=190)$. Participants with a previous colonoscopy within the past year, a history of CRC or other colorectal diseases, familial colorectal cancer syndromes, and past partial resection of the colon were excluded. Written informed consent was obtained from all study participants and institutional review boards at both Fred Hutchinson Cancer Research Center and Kaiser Permanente Washington approved the study.

\section{Data collection and case/control ascertainment}

Participants in both phases completed a structured questionnaire to collect information on risk factors, and medical, family, and colorectal cancer screening history. Biopsies taken at the time of index colonoscopy received a standardized pathology review, as described elsewhere [5]. Participants were diagnosed as having adenomas (tubular, villous or tubulovillous adenomas), serrated polyps (hyperplastic polyps, traditional serrated adenomas, or SSA/Ps), or both. Polyp-free controls were identified during index colonoscopy, and reflected the age distribution and diagnosis year of the polyp cases. Adenomas $\geq 10 \mathrm{~mm}$ in diameter or with $\geq 20 \%$ villous components or exhibiting high-grade dysplasia were classified as advanced adenomas (nonadvanced otherwise). SSA/Ps were considered as advanced lesions, while hyperplastic polyps were considered to be non-advanced lesions in the serrated polyps subgroup.

\section{Telomere length measurement}

Genomic DNA was extracted from lymphocytes (phase 1) or buccal DNA (phase 2) using the Qiagen QIAamp DNA extraction kit (Qiagen Inc., Valencia, CA) and stored at $80{ }^{\circ} \mathrm{C}$. Telomere length $(\mathrm{T})$ relative to a single copy gene 36B4 (S) was measured using a singleplex quantitative polymerase chain reaction ( $\mathrm{qPCR}$ ), as described by Cawthon [6]. All assays were run in triplicate and a standard laboratory control was run on each plate. Each plate run was then assessed for efficiency and precision (R2) using the standard curve generated using the laboratory control. R2 values for the telomere and control gene standard curves were greater than 0.98 , demonstrating great precision. Efficiency for the telomere and control gene PCRs were between $99 \%$ and $110 \%$. The mean normalized $\mathrm{T} / \mathrm{S}$ ratio was used for the statistical analysis.

\section{Statistical analyses}

Multivariable polytomous logistic regression was used to compare case groups (adenomas only, serrated polyps only, or both) with each other and with polyp-free controls. Odds ratios (OR) and 95\% confidence intervals (CIs) for short TL (shortest telomere tertile; $\mathrm{T} / \mathrm{S}$ ratio $<0.58$ ) were computed adjusting for age, sex, race, smoking status, body mass index, and use of anti-inflammatory drugs These factors were selected a priori based on evidence from prior studies reporting associations between them and TL or colorectal polyps. Wald $p$-values were calculated to compare heterogeneity between case groups. Analyses evaluating associations between TL and lesion severity included persons with synchronous adenomas and serrated polyps within both the adenoma as well as serrated pathway groups (Fig. 1), because we were interested in evaluating the differences between advanced and non-advanced lesions within a particular pathway, irrespective of the presence of polyp lesions from another pathway. All analyses were performed using Stata (v14.0; Stata Corp).

\section{Results}

Of the 190 study participants with available DNA specimens, 80 were classified as having adenomas, 33 as serrated polyps, 28 as having both adenomas and serrated polyps, and were compared to 49 polyp-free controls. Demographic comparisons between polyp subtype cases and controls are shown in Table 1. Participants with adenomas only were slightly older and more likely than controls to be male and former smokers. In contrast, study participants with serrated polyps only were more likely to be female, current/former smokers, and obese. Cases were similar to controls with respect to other lifestyle exposures such as their educational status, and anti-inflammatory drug use.

Table 2 presents the ORs and 95\% CI for each colorectal polyp subtype outcomes according to shortest telomere tertile. Having $\mathrm{TL}$ in the shortest tertile $(\mathrm{T} / \mathrm{S}$ ratio $<0.58)$ was associated with an increased risk of serrated polyps [adjusted OR $(95 \% \mathrm{CI})=2.98(1.15,7.77)]$ even after adjustment for confounding effects of age, sex, race, smoking status, body mass index, and use of anti-inflammatory drugs. Short TLs were also modestly and non-significantly associated with increased adenoma risk $[\mathrm{OR}(95 \% \mathrm{CI})=1.77$ $(0.81,3.88)]$ in adjusted models. When evaluated by lesion severity within each pathway, short telomeres appeared to be more strongly associated with advanced adenomas 


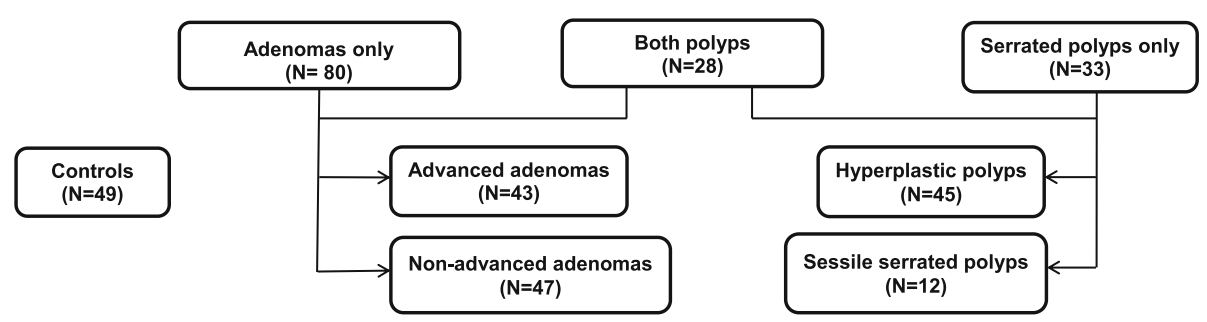

Fig. 1 Study participants' outcomes based on index colonoscopy

$[\mathrm{OR}(95 \% \mathrm{CI})=1.90(0.76,4.73)]$ and sessile serrated polyps $[\mathrm{OR}(95 \% \mathrm{CI})=3.82(0.86,16.86)]$, although these associations did not reach statistical significance. Lastly, we did not find any evidence for heterogeneity in the associations with short TL for adenomas vs. serrated polyps ( $p$ for heterogeneity $=0.42$ ) or for lesion severity comparisons within the adenoma and serrated pathways.

\section{Discussion}

There are limited prior studies evaluating the association between shortened telomeres and colorectal neoplasia. Two case-control studies nested within large cohorts observed a lack of association between TL and CRC [7-9]. Conversely, Pooley et al. have reported significantly shorter telomeres in circulating leukocytes among CRC cases than controls [10]. More recently, a large population-wide Danish study of over 47,000 individuals reported no association, while a Chinese study reported increased CRC risk with shorter TL $[9,11]$. Summarizing these findings, a very recent meta-analysis noted that the available evidence is insufficient to understand the exact role of telomere length in the development of colorectal cancer, and highlighted the need for future studies [12]. Fewer studies have evaluated the relationship between colorectal cancer precursor lesions and TL. A recent report suggested an increased risk for advanced adenomas in persons with short leukocyte TL [13]; however, this study was relatively small (35 advanced adenomas cases) and did not evaluate

Table 1 Participant characteristics by polyp status, Kaiser Permanente Washington, Seattle, Washington, 1998-2007

\begin{tabular}{|c|c|c|c|c|c|}
\hline Patient characteristics & & $\begin{array}{l}\text { Controls } \\
(n=49)\end{array}$ & $\begin{array}{l}\text { Adenomas } \\
(n=80)\end{array}$ & $\begin{array}{l}\text { Serrated polyps } \\
(n=33)\end{array}$ & $\begin{array}{l}\text { Both Adenomas + Serrated polyps } \\
(n=28)\end{array}$ \\
\hline Age in years; Mean (SD) & & $59.7(10.8)$ & $62.3(10.4)$ & $61.7(9.2)$ & $62.3(10.9)$ \\
\hline \multirow[t]{2}{*}{ Gender; n(\%) } & Male & $25(51)$ & $44(55)$ & $13(39.4)$ & $13(46.4)$ \\
\hline & Female & $24(49)$ & $36(45)$ & $20(60.6)$ & $15(53.6)$ \\
\hline White race; n(\%) & & $44(89.8)$ & $72(90)$ & $29(87.8)$ & $25(89.3)$ \\
\hline \multirow[t]{3}{*}{ Smoking; n(\%) } & Never & $20(40.8)$ & $27(33.8)$ & $9(27.3)$ & $9(32.1)$ \\
\hline & Former & $22(44.9)$ & $50(62.5)$ & $20(60.6)$ & $14(50)$ \\
\hline & Current & $7(14.3)$ & $3(3.8)$ & $4(12.1)$ & $5(17.9)$ \\
\hline Alcohol use; n(\%) & & $31(63.3)$ & $44(55)$ & $22(66.7)$ & $18(64.3)$ \\
\hline \multirow[t]{3}{*}{$\mathrm{BMl} ; \mathrm{n}(\%)$} & $<25$ & $17(34.7)$ & $25(31.3)$ & $10(30.3)$ & $8(28.6)$ \\
\hline & $25-29.99$ & 19 (38.8) & $37(46.3)$ & $10(30.3)$ & $9(32.1)$ \\
\hline & $30+$ & $13(26.5)$ & $17(21.4)$ & $13(39.4)$ & $11(39.3)$ \\
\hline \multirow[t]{3}{*}{ Education; n(\%) } & $<$ High school & $8(16.3)$ & $11(13.8)$ & $4(12.1)$ & $3(10.7)$ \\
\hline & Some college & $11(22.5)$ & $21(26.3)$ & $9(27.3)$ & $12(42.9)$ \\
\hline & College + & $30(61.2)$ & $48(60)$ & $20(60.6)$ & $13(46.4)$ \\
\hline Anti-inflammatory drug use; n(\%) & & $26(53.1)$ & $41(51.3)$ & $17(51.5)$ & $10(35.7)$ \\
\hline Physical activity $\geq 60$ MET h/wk.; n(\%) & & $41(83.7)$ & $62(77.5)$ & $26(78.8)$ & $23(82.1)$ \\
\hline Relative Telomere length; Median (IQR) & & $0.60(0.56-0.72)$ & $0.60(0.50-0.71)$ & $0.57(0.45-0.67)$ & $0.59(0.51-0.67)$ \\
\hline Short telomeres; n(\%) & $\begin{array}{l}\mathrm{T} / \mathrm{S}<0.58 \text { or } \\
\text { shortest tertile }\end{array}$ & $17(34.7)$ & $36(45.6)$ & $19(57.8)$ & $14(50)$ \\
\hline
\end{tabular}


Table 2 Associations between shortest telomere tertile and colorectal polyp subtypes

\begin{tabular}{|c|c|c|c|c|}
\hline & \multirow{3}{*}{$\begin{array}{l}\text { Telomere tertile }^{\mathrm{a}} 2 \& 3 \\
\text { T/S ratio }>=0.58 \\
N(\%)\end{array}$} & \multicolumn{3}{|c|}{ Telomere tertile 1} \\
\hline & & \multicolumn{3}{|l|}{$\mathrm{T} / \mathrm{S}$ ratio $<0.58$} \\
\hline & & $N(\%)$ & Unadjusted OR & Adjusted $^{\mathrm{b}} \mathrm{OR}$ \\
\hline \multicolumn{5}{|l|}{ Adenoma-Serrated Outcome } \\
\hline Controls & $32(65.3)$ & $17(34.7)$ & REF & REF \\
\hline Adenomas & $43(54.3)$ & $36(45.6)$ & $1.58(0.75,3.29)$ & $1.77(0.81,3.88)$ \\
\hline Serrated & $14(42.4)$ & $19(57.6)$ & $2.55(1.03,6.33)$ & $2.98(1.15,7.77)$ \\
\hline Both & $14(50.0)$ & $14(50.0)$ & $1.88(0.73,4.85)$ & $1.94(0.72,5.27)$ \\
\hline \multicolumn{5}{|c|}{$P$ for heterogeneity between Adenomas \& Serrated $=0.42$} \\
\hline \multicolumn{5}{|l|}{ Adenoma Outcome } \\
\hline Controls & $32(65.3)$ & $17(34.7)$ & REF & REF \\
\hline Non-advanced adenoma & $25(54.4)$ & $21(45.7)$ & $1.58(0.69,3.61)$ & $1.63(0.69,3.86)$ \\
\hline Advanced adenoma & $22(51.2)$ & $21(48.8)$ & $1.80(0.78,4.16)$ & $1.90(0.76,4.73)$ \\
\hline \multicolumn{5}{|c|}{ P for heterogeneity between Advanced \& Non-advanced adenomas $=0.71$} \\
\hline \multicolumn{5}{|l|}{ Serrated Outcome } \\
\hline Controls & $32(65.3)$ & $17(34.7)$ & REF & REF \\
\hline Hyperplastic polyps & $22(48.9)$ & $23(51.1)$ & $1.97(0.86,4.51)$ & $2.24(0.88,5.70)$ \\
\hline Sessile serrated polyps & $4(33.3)$ & $8(66.7)$ & $3.76(0.99,14.3)$ & $3.82(0.86,16.86)$ \\
\hline \multicolumn{5}{|c|}{ P for heterogeneity between Hyperplastic \& Sessile Serrated Polyps $=0.61$} \\
\hline
\end{tabular}

a Telomere tertiles were based on the telomere length distribution among controls only

${ }^{\mathrm{b}}$ Models adjusted for age, gender, race, BMI, smoking, and use of anti-inflammatory drugs

the relationship with serrated polyps. Roger et al. reported in an experimental setting that extensive tissue telomere erosion may lead to chromosomal instability and initiation of CRC in polyps from familial adenomatous polyposis patients [14]. The authors hypothesize that this may be a potential mechanism resulting in chromosomal instability and malignant transformation. Another cross-sectional study found that TL in large adenoma lesions $(>2 \mathrm{~cm})$ was significantly shorter than normal surrounding tissue from the same individuals [15]. Taken together, these studies suggest that telomere shortening may play a role in the development of both colorectal polyps and/or CRC. However, further studies are required to better understand the role of TL (and the point at which they may act) in the pathological progression in colorectal neoplasia.

Our analysis is an important addition to the literature, because it is the first study to report a statistically significant association of TL with serrated polyps, suggesting that telomeres may play an important role along the entire serrated pathway. There was also a suggestion of increased risk, particularly, for SSA/Ps. SSA/Ps are newly-recognized precursors to CRC [16]; thus, this finding may provide valuable insights into the underlying mechanisms for cancer progression within the serrated pathway. Further work is warranted to determine whether TL can serve as a reliable biomarker for such a neoplastic progression.
Our study is larger than most other studies of colorectal polyps and TL, but it is nonetheless limited by sample size, particularly as colorectal polyp cases were further divided into smaller subgroups based on lesion severity. However, this enabled us to look at differences within sub-classes of colorectal polyps. Also, misclassification of disease status in some cases and controls is possible, particularly with respect to the harder to visualize SSA/Ps. However, such misclassification would be non-differential and would bias estimates towards the null. Hence, our reported positive association with serrated polyps is conservative. Finally, although we have controlled for major potential confounders, including age, sex, smoking, body mass index, and inflammation, we cannot exclude the possibility of residual confounding by measured and unmeasured risk factors. Strengths of our study include the high-quality and detailed characterization of colorectal polyp cases and controls using colonoscopy and standardized pathology review, which enabled us to evaluate the relationship between short telomeres and various polyp subtypes. Additionally, to our knowledge, this is the first study to report associations between short telomeres and advanced polyps within the serrated pathway.

\section{Conclusions}

In conclusion, short telomeres were associated with an increased risk of colorectal polyps in both the 
adenoma-carcinoma and serrated pathways, although the increased risk was statistically significant only for serrated polyps. Among individuals with serrated polyps, the risk associated with short telomeres appeared stronger, but not statistically significant, for those with SSA/Ps. Future prospective studies are needed to define the temporal sequence between TL and these high risk lesions, and to elucidate the role of telomeres as a biomarker for CRC risk stratification.

\section{Abbreviations}

Cl: Confidence interval; CRC: Colorectal cancer; OR: Odds ratio; SSA/P: Sessile serrated adenoma/polyp; TL: Telomere length

\section{Acknowledgements}

We thank the late Dr. Jeremy Jass for his many contributions in the early stages of this research. We acknowledge the members of the Molecular Epidemiology Laboratory at the Fred Hutchinson Cancer Research Center for the telomere length assays.

\section{Funding}

This work was supported by the National Cancer Institute grant P01 CA074184 (J. D. Potter \& P. A. Newcomb) and R01 CA097325 (P.A. Newcomb). Additionally, National Cancer Institute grants R25CA094880 (S. Hardikar), K05CA152715 (P. A. Newcomb), KL2 TR000421 (A.N. Burnett-Hartman) and K07CA172298 (A.I. Phipps) have also supported this work. The funders/ sponsors had no role in the design and conduct of the study; collection, management, analysis, and interpretation of the data; preparation, review, or approval of the manuscript; and decision to submit the manuscript for publication.

\section{Availability of data and materials}

The datasets used and/or analyzed during the current study will be available from the corresponding author on reasonable request.

\section{Authors' contributions}

Study concept and design: SH, PAN, ANB, AIP. Acquisition, analysis, or interpretation of data: SH, ANB, AIP, MPU, LZ, PAN. Critical revisions of the manuscript: SH, ANB, AIP, MPU, LZ, PAN. Study supervision: SH, PAN. All the authors have read and approved the final manuscript.

\section{Ethics approval and consent to participate}

Institutional review boards at both Fred Hutchinson Cancer Research Center and Kaiser Permanente Washington approved the study protocols. All study participants provided written informed consent at the time of enrollment into the study.

\section{Competing interests}

The authors declare that they have no competing interests.

\section{Publisher's Note}

Springer Nature remains neutral with regard to jurisdictional claims in published maps and institutional affiliations.

\section{Author details}

${ }^{1}$ Huntsman Cancer Institute, University of Utah, 2000 Circle of Hope Dr. Room 4711, Salt Lake City, UT 84112, USA. ² Public Health Sciences Division, Fred Hutchinson Cancer Research Center, Seattle, WA, USA. ${ }^{3}$ Kaiser Permanente, Colorado Institute for Health Research, Denver, CO, USA ${ }^{4}$ Department of Epidemiology, School of Public Health, University of Washington, Seattle, WA, USA. ${ }^{5}$ Department of Pathology, School of Medicine, University of Washington, Seattle, WA, USA. ${ }^{6}$ Kaiser Permanente Washington Health Research Institute, Seattle, WA, USA.
Received: 25 July 2017 Accepted: 23 April 2018

Published online: 02 May 2018

\section{References}

1. Jass JR. Molecular heterogeneity of colorectal cancer: implications for cancer control. Surg Oncol. 2007;16 Suppl 1:S7-9. https://doi.org/10.1016/j.suronc. 2007.10.039

2. Regula J, Rupinski M, Kraszewska E, Polkowski M, Pachlewski J, Orlowska J, et al. Colonoscopy in colorectal-cancer screening for detection of advanced neoplasia. N Engl J Med. 2006;355(18):1863-72. https://doi.org/10.1056/ NEJMoa054967.

3. Wong JM, Collins K. Telomere maintenance and disease. Lancet. 2003; 362(9388):983-8. https://doi.org/10.1016/S0140-6736(03)14369-3.

4. Wentzensen IM, Mirabello L, Pfeiffer RM, Savage SA. The association of telomere length and cancer: a meta-analysis. Cancer Epidemiol Biomarkers Prev. 2011;20(6):1238-50. https://doi.org/10.1158/1055-9965. EPI-11-0005; 10. 1158/1055-9965.EPI-11-0005

5. Burnett-Hartman AN, Passarelli MN, Adams SV, Upton MP, Zhu LC, Potter JD, et al. Differences in epidemiologic risk factors for colorectal adenomas and serrated polyps by lesion severity and anatomical site. Am J Epidemiol. 2013;177(7):625-37. https://doi.org/10.1093/aje/kws282.

6. Cawthon RM. Telomere measurement by quantitative PCR. Nucleic Acids Res. 2002;30(10):e47

7. Zee RY, Castonguay AJ, Barton NS, Buring JE. Mean telomere length and risk of incident colorectal carcinoma: a prospective, nested case-control approach. Cancer Epidemiol Biomarkers Prev. 2009;18(8):2280-2. https://doi. org/10.1158/1055-9965.epi-09-0360.

8. Lee IM, Lin J, Castonguay AJ, Barton NS, Buring JE, Zee RY. Mean leukocyte telomere length and risk of incident colorectal carcinoma in women: a prospective, nested case-control study. Clin Chem Lab Med. 2010;48(2):259-62. https://doi.org/10.1515/cclm.2010.049.

9. Weischer M, Nordestgaard BG, Cawthon RM, Freiberg JJ, Tybjaerg-Hansen A, Bojesen SE. Short telomere length, cancer survival, and cancer risk in 47102 individuals. J Natl Cancer Inst. 2013;105(7):459-68. https://doi.org/10.1093/ jnci/djt016.

10. Pooley KA, Sandhu MS, Tyrer J, Shah M, Driver KE, Luben RN, et al. Telomere length in prospective and retrospective cancer case-control studies. Cancer Res. 2010;70(8):3170-6. https://doi.org/10.1158/0008-5472. CAN-09-4595; 10. 1158/0008-5472.CAN-09-4595

11. Qin Q, Sun J, Yin J, Liu L, Chen J, Zhang Y, et al. Telomere length in peripheral blood leukocytes is associated with risk of colorectal cancer in Chinese population. PLoS One. 2014;9(2):e88135. https://doi.org/10.1371/ journal.pone.0088135.

12. Naing C, Aung K, Lai PK, Mak JW. Association between telomere length and the risk of colorectal cancer: a meta-analysis of observational studies. BMC Cancer. 2017:17(1):24. https://doi.org/10.1186/s12885-016-2997-3.

13. Riegert-Johnson DL, Boardman LA, Crook JE, Thomas CS, Johnson RA, Roberts ME. Shorter peripheral blood telomeres are a potential biomarker for patients with advanced colorectal adenomas. Int J Biol Markers. 2012; 27(4):e375-80. https://doi.org/10.5301/JBM.2012.9347.

14. Roger L, Jones RE, Heppel NH, Williams GT, Sampson JR, Baird DM. Extensive telomere erosion in the initiation of colorectal adenomas and its association with chromosomal instability. J Natl Cancer Inst. 2013;105(16):1202-11. https://doi.org/10.1093/jnci/djt191.

15. O'Sullivan J, Risques RA, Mandelson MT, Chen L, Brentnall TA, Bronner MP, et al. Telomere length in the colon declines with age: a relation to colorectal cancer? Cancer Epidemiol Biomarkers Prev. 2006;15(3):573-7. https://doi.org/10.1158/1055-9965.epi-05-0542.

16. Erichsen R, Baron JA, Hamilton-Dutoit SJ, Snover DC, Torlakovic EE, Pedersen $L$ et al. Increased risk of colorectal Cancer development among patients with serrated polyps. Gastroenterology. 2016:150(4):895-902.e5. https://doi. org/10.1053/j.gastro.2015.11.046. 